\title{
Field-Based Scattering-Matrix Extraction Scheme for the FVTD Method Exploiting a Flux-Splitting Algorithm
}

\author{
Dirk Baumann, Student Member, IEEE, Christophe Fumeaux, Member, IEEE, and Rüdiger Vahldieck, Fellow, IEEE
}

\begin{abstract}
This paper introduces a novel field-based scheme for the extraction of a generalized-scattering matrix. A flux-splitting (FS) algorithm that separates the total electromagnetic field into an incident and a reflected part is used for a new definition of power waves. This flux-separation scheme, based on a plane-wave approach of the split waves, originates from finite-volume techniques. However, it can be applied locally in any numerical scheme that uses a volume discretization. To yield correct $S$-parameters, the FS matrix needs to be adapted through introduction of modified wave velocities to match the plane-wave condition. The introduced extraction scheme is applied to the finite-volume time-domain method and compared successfully to reference solutions for TEM and non-TEM structures, both with homogeneous and inhomogeneous cross sections.
\end{abstract}

Index Terms-Finite volume time domain (FVTD), flux splitting (FS), scattering parameters.

\section{INTRODUCTION}

$\mathbf{T}$ HE finite-volume time-domain (FVTD) method is a promising numerical technique with great potential for the simulation of complex electromagnetic problems. Although still quite new in computational electromagnetics, FVTD has already been demonstrated as being applicable for a wide range of problems [1]-[3]. Yet the full potential of FVTD, however, still remains to be unfolded. To pave the way for a broader application range of FVTD, comparable to that of the finite-difference time-domain (FDTD) method, several problems need to be addressed. Among those is the issue of $S$-parameter extraction in single and multimode problems. $S$-matrix determination encounters several difficulties in FVTD since the method relies on unstructured inhomogeneous meshes and, therefore, the $S$-matrix, for example, of a waveguide discontinuity is not easily determined.

In this paper, a novel field-based approach for the extraction of a generalized scattering matrix is proposed. The presented scheme exploits the flux-splitting (FS) algorithm that was introduced for the FVTD method by Shankar et al. [4]. The FVTD method is applied in unstructured inhomogeneous meshes using polyhedral cells to approximate the modeled geometry with a high accuracy. The interaction between two adjacent elementary cells is characterized by mathematically defined fluxes through their shared face. These fluxes can be separated into outgoing

Manuscript received April 4, 2005; revised June 29, 2005.

The authors are with the Laboratory for Electromagnetic Fields and Microwave Electronics, Swiss Federal Institute of Technology Zürich, CH-8092 Zürich, Switzerland (e-mail: fvtd@ifh.ee.ethz.ch).

Digital Object Identifier 10.1109/TMTT.2005.857103 and incoming parts, which, for the computation of scattering parameters, are connected to the incident and reflected waves in a port plane [5].

Although the FS scheme originates from a finite-volume algorithm, its application is not restricted to this technique. The matrix needed for the flux separation can be set up independently of the numerical method on the basis of the electromagnetic fields obtained in the simulation. Therefore, following the FS procedure proposed here, the scattering parameters can be extracted in any numerical method that is based on a volumediscretizing scheme.

In the following, the $S$-parameter extraction scheme is incorporated into the FVTD method [6], which is briefly reviewed in Section II. A problem introduced by the unstructured mesh of the FVTD is that port planes generally do not coincide with cell boundaries, for example, as in the FDTD method. The question of port definition is then addressed in Section III.

The basic idea of extracting the generalized scattering matrix can be described in the following three parts:

- FS algorithm that constitutes the basis of the proposed scheme (Section IV);

- mode-orthogonality relation needed for the extraction of a generalized $S$-matrix (Section V);

- power wave extraction yielding the scattering parameters (Section VI).

The flux-separation scheme assumes local plane waves propagating through the elementary cell faces. To assure a correct power density of incident and reflected waves in a port for $S$-parameter extraction, a modified wave velocity of the electric and magnetic fields is introduced in the FS matrix. For validation purposes, the presented field-based extraction scheme is compared to a transmission-line-based approach relying on voltage and current (UI). This approach is borrowed from FDTD [7] and implemented here in the FVTD method.

Finally, the FS-based scheme is applied successfully to the extraction of scattering parameters. Four examples are shown. First, a coaxial-torus resonator is investigated, showing the accurate results obtained for TEM structures. Second, the return loss of a circular corrugated horn antenna is computed as an example for non-TEM and radiating structures. Third, a waveguide exhibiting an $H$-plane discontinuity is analyzed and the generalized scattering parameters are presented. Fourth, a waveguide filter loaded with dielectric slabs is analyzed to demonstrate the applicability of the proposed scheme also in structures incorporating inhomogeneous cross sections. In all examples, 
the FVTD results are compared to solutions from established numerical methods.

\section{FVTD METHOD}

The FVTD method originates from computational fluid dynamics and has been adapted for electromagnetics at the end of the 1980s [4], [8]. Implementations of the FVTD method are based on the Maxwell's curl equations that are integrated over a finite volume. Here, tetrahedral elementary cells (four faces) are used. In a discretized form, these integrals for the elementary cell $i$ with the volume $V_{i}$ can be written as

$$
\frac{\partial \boldsymbol{U}_{i}}{\partial t}=-\frac{1}{V_{i}} \sum_{k=1}^{4} \boldsymbol{\Phi}_{k} F_{k}
$$

where $\boldsymbol{U}_{i}=\left(E_{i x}, E_{i y}, E_{i z}, H_{i x}, H_{i y}, H_{i z}\right)^{T}$ is a vector containing the electric and magnetic fields in the barycenter of cell $i$. $\boldsymbol{\Phi}_{k}$ can be interpreted as a flux through the triangular cell face $k$ with the area $F_{k}$ and the outward-pointing normal vector $\boldsymbol{n}_{k}$. The flux is connected to the electromagnetic field by

$$
\boldsymbol{\Phi}_{k}=\left(\begin{array}{c}
-\boldsymbol{n}_{k} \times \frac{1}{\varepsilon} \boldsymbol{H}_{k} \\
\boldsymbol{n}_{k} \times \frac{1}{\mu} \boldsymbol{E}_{k}
\end{array}\right)
$$

where $\boldsymbol{E}_{k}$ and $\boldsymbol{H}_{k}$ are field values on the triangular face $k$. From (2), it can be seen that the flux $\boldsymbol{\Phi}_{k}$ only contains field components tangential to the face $k$.

The linear dimension of the tetrahedral cells in the volume mesh is typically specified by a tenth of the wavelength at the highest frequency of interest. When small structural details are resolved, the size of the elementary cells in those regions is reduced accordingly. The necessity to use a single short time step imposed by the smallest cells in the mesh is abolished using geometry-matched local-time steps [9]. A Lax-Wendroff predictor-corrector upwind scheme is used for time discretization providing a second-order accuracy in time. Performing an eigendecomposition and using a local plane-wave approach, an FS algorithm can be applied. For each triangular face, only plane waves propagating in normal direction $\boldsymbol{n}_{k}$ are considered [6]. The total flux is then separated into an outgoing (superscript + ) and an incoming (superscript -) part

$$
\Phi_{k}=\Phi_{i k}^{+}+\Phi_{j k}^{-}
$$

where: 1) the outgoing flux $\boldsymbol{\Phi}_{i k}^{+}$is determined on the basis of the fields $\boldsymbol{U}_{i}$ inside the actual cell $i$ and 2) the incoming flux $\boldsymbol{\Phi}_{j k}^{-}$is calculated using the field $\boldsymbol{U}_{j}$ in the neighboring cell $j$. The fields $\boldsymbol{U}_{i k}$ and $\boldsymbol{U}_{j k}$ on both sides of the face $k$, which are required for the computation of the fluxes, are determined by a monotonic upwind scheme for conservation laws (MUSCL) introducing a second-order accuracy in space [6].

The separation of fluxes into an outgoing and an incoming part can be used for the implementation of a Silver-Müller absorbing boundary condition (ABC), where the incoming fluxes are forced to zero as follows:

$$
\Phi_{j k}^{-}=0 .
$$

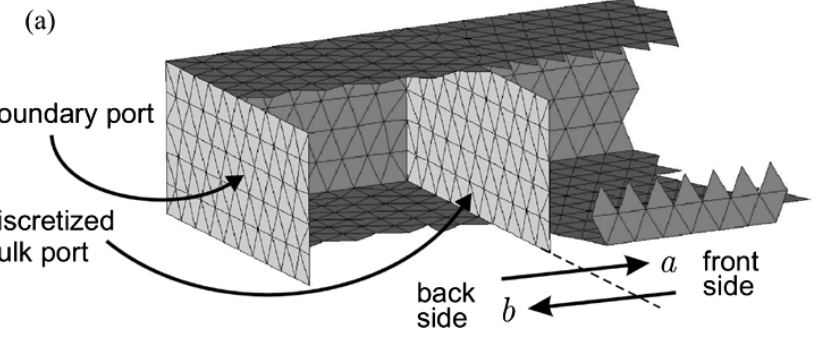

(b)

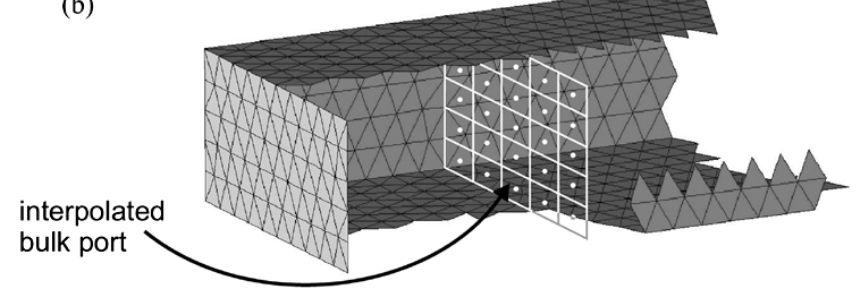

Fig. 1. Inserting: (a) discretized and (b) interpolated port planes in the unstructured mesh. Port planes can be either located on the computational boundary (boundary port) or in the computational bulk (bulk port). The definition of back and front sides of a port determines the direction of incident $a$ and reflected $b$ wave.

The exploitation of the FS algorithm for the extraction of a generalized scattering matrix is described in Section VI. The scheme connects the separated outgoing and incoming fluxes to the incident and reflected waves in a port.

\section{PORT DEFINITION}

A port plane, used for the extraction of scattering parameters, must provide a reference position to enable an unambiguous determination of the phase of incident and reflected waves. In methods using structured meshes, e.g., cubic cells in FDTD, the sidewalls of the elementary cells may be used to build a port reference plane that is perpendicular to the direction of wave propagation. In FVTD, special care must be taken to define port planes since the method is applied in unstructured inhomogeneous meshes. In general, the tetrahedral cells themselves do not form a planar surface and, therefore, an artificial port plane has to be introduced in the model. Such a plane can be realized in two different ways, i.e., either as a discretized surface embedded in the mesh or by an interpolation procedure of the electromagnetic field onto a predefined imaginary regular grid. Both possibilities, implemented and tested successfully, are explained in Sections III-A and B.

\section{A. Discretized Port Plane}

In order to force the tetrahedral elementary cells in the mesh to be aligned at one side and, thus, to form a reference plane, an artificial port plane can be introduced in the meshed model. This plane is incorporated perpendicularly to the propagation direction of the waveguide and meshed with triangles. Such a plane can be either located on the boundary of the computational domain (boundary port) or inside the computational bulk (bulk port), as depicted in Fig. 1(a). Boundary ports can additionally be employed as: 1) source planes impressing a certain field distribution as incoming fluxes and 2) an $\mathrm{ABC}$, where the incoming fluxes are forced to zero preventing unwanted reflections from the boundary. For the extraction of the scattering pa- 


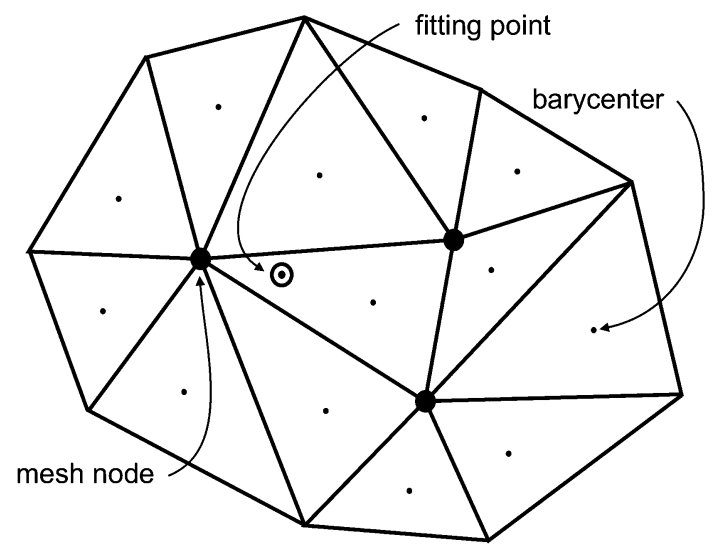

Fig. 2. 2-D representation of the neighborhood of a fitting point used for the fitting procedure.

rameters, field values on both sides of a port plane are used. For bulk ports, these values are obtained from the electromagnetic fields in the barycenters of the adjacent tetrahedra. In the case of boundary ports, impressed fields (sources) are substituting the barycenter values on the back side of the port.

\section{B. Interpolated Port Plane}

Alternatively to a discretized plane, ports can be defined by interpolation points on a regular planar grid [see Fig. 1(b)]. Field values in the barycenter of the neighboring tetrahedral cells are used as data for interpolation. The output of the FVTD algorithm yields the field values in the barycenter of these tetrahedra. From these three-dimensional (3-D) scattered FVTD data, two local methods have been tested for extracting field data on two-dimensional (2-D) port grids, which are: 1) a linear fitting with quadratic functions and 2) an inverse-distance weighted Shepard method (improved using spatial derivatives) [10]. Judging the quality of the interpolation results is a difficult problem that requires a somewhat subjective judgment. Considering the smoothness and visual appearance of the obtained results, the linear fitting with quadratic functions seems to yield better results than the Shepard interpolation procedure in the context of the FVTD tetrahedral mesh.

To perform the local fitting procedure for a given point, field values in the barycenters of the cells in the neighborhood of this fitting point are used. In the tetrahedral mesh, those neighboring cells of a fitting point that are accounted for fitting are determined by the following procedure.

Step 1) Determine the tetrahedron in which the fitting point is located.

Step 2) Find all tetrahedra that share at least one node with the starting tetrahedron.

Step 3) Perform a material and boundary check to exclude any discontinuities, for example, such as perfect electric conductor (PEC) boundaries located in between the fitting point and the neighboring tetrahedron.

Fig. 2 shows an example of the definition of the neighborhood of a fitting point in a 2-D representation, i.e., using triangles instead of tetrahedra. In three dimensions, typically $20-25$ tetrahedra share a single node.

\section{Field-Based Wave Separation}

Starting from Maxwell's equations in a conservative form [6], the flux through the face $k$, as defined in (2), can be written as

$$
\boldsymbol{\Phi}_{k}=\boldsymbol{F}\left(\boldsymbol{n}_{k}\right) \boldsymbol{U}_{k}
$$

where $\boldsymbol{n}_{k}=\left(n_{k x}, n_{k y}, n_{k z}\right)^{T}$ is the outwards-pointing normal vector of the considered cell face $k$. The matrix $\boldsymbol{F}\left(\boldsymbol{n}_{k}\right)$ is given by

$$
\left(\begin{array}{cccccc}
0 & 0 & 0 & 0 & \frac{n_{k z}}{\varepsilon} & -\frac{n_{k y}}{\varepsilon} \\
0 & 0 & 0 & -\frac{n_{k z}}{\varepsilon} & 0 & \frac{n_{k x}}{\varepsilon} \\
0 & 0 & 0 & \frac{n_{k y}}{\varepsilon} & -\frac{n_{k x}}{\varepsilon} & 0 \\
0 & -\frac{n_{k z}}{\mu} & \frac{n_{k y}}{\mu} & 0 & 0 & 0 \\
\frac{n_{k z}}{\mu} & 0 & -\frac{n_{k x}}{\mu} & 0 & 0 & 0 \\
-\frac{n_{k y}}{\mu} & \frac{n_{k x}}{\mu} & 0 & 0 & 0 & 0
\end{array}\right)
$$

exhibiting the six real eigenvalues

$$
(0,0, c, c,-c,-c)
$$

where $c=1 / \sqrt{\varepsilon \mu}$ is the velocity of light in the medium. Using these eigenvalues, the matrix $\boldsymbol{F}\left(\boldsymbol{n}_{k}\right)$ can be split into a positive and a negative part

$$
\boldsymbol{F}\left(\boldsymbol{n}_{k}\right)=\boldsymbol{F}\left(\boldsymbol{n}_{k}\right)^{+}+\boldsymbol{F}\left(\boldsymbol{n}_{k}\right)^{-} .
$$

The matrix $\boldsymbol{F}\left(\boldsymbol{n}_{k}\right)^{+}$is given in (13), shown at the bottom of the following page, where the following abbreviations are used:

$$
\begin{aligned}
& a_{1}=n_{k x}^{2}+n_{k y}^{2} \\
& a_{2}=n_{k x}^{2}+n_{k z}^{2} \\
& a_{3}=n_{k y}^{2}+n_{k z}^{2} \\
& a_{4}=n_{k x} n_{k y} \\
& a_{5}=n_{k x} n_{k z} \\
& a_{6}=n_{k y} n_{k z} .
\end{aligned}
$$

By applying matrix (8), the flux through a face of a cell in the computational mesh can be separated into an outgoing and incoming part. The outgoing part is connected to the positive eigenvalues of matrix $\boldsymbol{F}\left(\boldsymbol{n}_{k}\right)$ and is, therefore, denoted by superscript + . The matrix $\boldsymbol{F}\left(\boldsymbol{n}_{k}\right)^{-}$, associated to the incoming wave, can be expressed from the outgoing matrix according to

$$
\boldsymbol{F}\left(\boldsymbol{n}_{k}\right)^{-}=-\boldsymbol{F}\left(-\boldsymbol{n}_{k}\right)^{+} .
$$

The outgoing flux into cell $i$ and the incoming flux from cell $j$, both through face $k$, are then determined using the separated matrix $\boldsymbol{F}\left(\boldsymbol{n}_{k}\right)$

$$
\begin{aligned}
\boldsymbol{\Phi}_{i k}^{+} & =\boldsymbol{F}\left(\boldsymbol{n}_{k}\right)_{i}^{+} \cdot \boldsymbol{U}_{i k} \\
\boldsymbol{\Phi}_{j k}^{-} & =\boldsymbol{F}\left(\boldsymbol{n}_{k}\right)_{j}^{-} \cdot \boldsymbol{U}_{j k}
\end{aligned}
$$




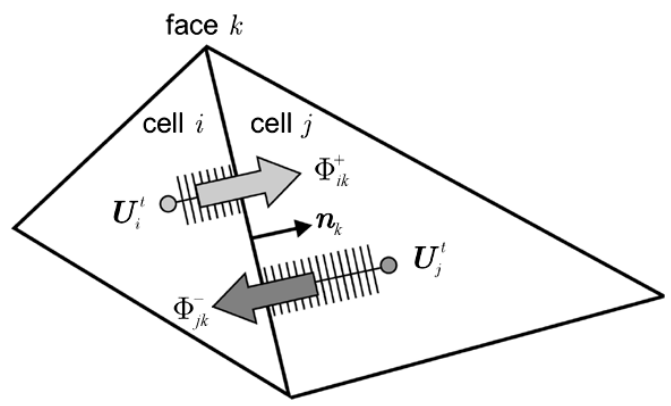

Fig. 3. 2-D representation of two adjacent cells $i$ and $j$. The outgoing and incoming fluxes, defined with respect to cell $i$, are determined on the basis of the field values in the barycenters of cell $i$ and cell $j$, respectively. The flux separation is based on a plane-wave assumption in direction of the normal vector $\boldsymbol{n}_{k}$.

where $\boldsymbol{U}_{i k}$ and $\boldsymbol{U}_{j k}$ are denoting the fields on the face $k$, computed on the basis of the barycenter field values of cell $i$, and $j$, respectively. Fig. 3 shows a 2-D representation of two adjacent cells, where the outgoing and incoming fluxes, defined with respect to cell $i$, are indicated by the light and dark gray arrows. It is important to note that the flux is separated on the basis of the assumption that plane waves are traveling through the cell face $k$ in the direction of the normal vector $\boldsymbol{n}_{k}$. Hence, the outgoing and incoming fluxes only contain information about the tangential-field values on the triangular face

$$
\boldsymbol{\Phi}_{i k}^{+}=\left(\begin{array}{c}
\boldsymbol{\Phi}_{i k}^{H+} \\
\boldsymbol{\Phi}_{i k}^{E+}
\end{array}\right)=\left(\begin{array}{c}
-\boldsymbol{n}_{k} \times \frac{1}{\varepsilon} \boldsymbol{H}_{i k}^{+} \\
\boldsymbol{n}_{k} \times \frac{1}{\mu} \boldsymbol{E}_{i k}^{+}
\end{array}\right)
$$

and

$$
\boldsymbol{\Phi}_{j k}^{-}=\left(\begin{array}{c}
\boldsymbol{\Phi}_{j k}^{H-} \\
\boldsymbol{\Phi}_{j k}^{E-}
\end{array}\right)=\left(\begin{array}{c}
-\boldsymbol{n}_{k} \times \frac{1}{\varepsilon} \boldsymbol{H}_{j k}^{-} \\
\boldsymbol{n}_{k} \times \frac{1}{\mu} \boldsymbol{E}_{j k}^{-}
\end{array}\right) .
$$

Knowing the outgoing and incoming fluxes through a face, the tangential fields on this face can be reconstructed from the fluxes. This fact is exploited for the extraction of the scattering parameters to compute the tangential fields of the incident and reflected waves in a port plane.

\section{ORTHOGONALITY OF MODES}

The total field in a waveguide can be represented by a superposition of single modes $p$ decomposed into transversal and longitudinal parts [11]. For the extraction of scattering parameters, only the tangential fields in the port plane are of interest.
Thus, for example, the total time-dependent tangential electric field at position $k$ can be expressed as [12]

$$
\boldsymbol{E}_{t}(k, t)=\sum_{p} A_{p}^{\prime}(t) \cdot \boldsymbol{e}_{p}^{\prime}(k)
$$

where $A_{p}^{\prime}(t)$ is the time-dependent amplitude of mode $p$ and $\boldsymbol{e}_{p}^{\prime}(k)$ is the mode-template vector at position $k$ containing the tangential-field distribution of mode $p$. In a port plane, the position $k$ corresponds to either a center of a triangular face [see Fig. 1(a)] or to a fitting point location [see Fig. 1(b)]. In guides with an homogeneous cross section, the following orthogonality relation is valid:

$$
\sum_{k=1}^{K}\left(\boldsymbol{e}_{q}^{\prime}(k) \cdot \boldsymbol{e}_{p}^{\prime}(k)\right) F_{k}= \begin{cases}0, & q \neq p \\ 1, & q=p\end{cases}
$$

where $F_{k}$ is the area of the face $k$ on the port plane. For a discretized port plane, $F_{k}$ is given by the area of the triangular face, and in the case of an interpolation port, by the ratio of the total area of the port plane to the number of interpolation points.

The mode-template vector has to be known a priori either analytically or from an eigenmode analysis at all $K$ points of a port plane. In order to determine the amplitude of a certain mode $p$, the total field and mode-template vector are used as follows:

$$
A_{p}^{\prime}(t)=\sum_{k=1}^{K}\left(\boldsymbol{E}_{t}(k, t) \cdot \boldsymbol{e}_{p}^{\prime}(k)\right) F_{k} .
$$

Similar expressions to (16)-(18) can be found for the $H$-field. However, they are not necessary since, for the extraction of the generalized $S$-matrix, only the electric-field values are used.

The above shown mode projection (18) relies on orthogonality of the modes and is valid only for TE and TM modes in homogeneous guides. For hybrid modes in inhomogeneously filled guides, the following orthogonality relation holds [13], [14]:

$$
\sum_{k=1}^{K}\left(\boldsymbol{e}_{q}^{\prime \prime}(k) \times \boldsymbol{h}_{p}^{\prime \prime}(k)\right) \boldsymbol{F}_{k}= \begin{cases}0, & q \neq p \\ 1, & q=p\end{cases}
$$

where $\boldsymbol{e}_{q}^{\prime \prime}(k)$ and $\boldsymbol{h}_{p}^{\prime \prime}(k)$ are the mode template vectors. Thus, the mode amplitude can be found by

$$
A_{p}^{\prime \prime}(t)=\sum_{k=1}^{K}\left(\boldsymbol{E}_{t}(k, t) \times \boldsymbol{h}_{p}^{\prime \prime}(k)\right) \boldsymbol{F}_{k} .
$$

$$
\boldsymbol{F}\left(\boldsymbol{n}_{k}\right)^{+}=\left(\begin{array}{l}
\boldsymbol{F}\left(\boldsymbol{n}_{k}\right)_{x}^{H^{+}} \\
\boldsymbol{F}\left(\boldsymbol{n}_{k}\right)_{y}^{H^{+}} \\
\boldsymbol{F}\left(\boldsymbol{n}_{k}\right)_{z}^{H^{+}} \\
\boldsymbol{F}\left(\boldsymbol{n}_{k}\right)_{x}^{E^{+}} \\
\boldsymbol{F}\left(\boldsymbol{n}_{k}\right)_{y}^{E^{+}} \\
\boldsymbol{F}\left(\boldsymbol{n}_{k}\right)_{z}^{E^{+}}
\end{array}\right)=\frac{1}{2}\left(\begin{array}{cccccc}
a_{3} c & -a_{4} c & -a_{5} c & 0 & n_{k z} / \varepsilon & -n_{k y} / \varepsilon \\
-a_{4} c & a_{2} c & -a_{6} c & -n_{k z} / \varepsilon & 0 & n_{k x} / \varepsilon \\
-a_{5} c & -a_{6} c & a_{1} c & n_{k y} / \varepsilon & -n_{k x} / \varepsilon & 0 \\
0 & -n_{k z} / \mu & n_{k y} / \mu & a_{3} c & -a_{4} c & -a_{5} c \\
n_{k z} / \mu & 0 & -n_{k x} / \mu & -a_{4} c & a_{2} c & -a_{6} c \\
-n_{k y} / \mu & n_{k x} / \mu & 0 & -a_{5} c & -a_{6} c & a_{1} c
\end{array}\right)
$$


Obviously, if the wave impedance is constant over the cross section of a port, (18) and (20) are equivalent apart from a constant, namely, the wave impedance. It is interesting to note that even in the case of an inhomogeneous waveguide loaded with a dielectric $\left(\varepsilon_{r}, \mu_{0}\right),(18)$ holds if the hybrid mode degenerates to a TE mode.

The above shown procedure of mode projection can be exploited both for minimizing numerical noise and for the extraction of generalized scatting parameters in a multimode environment.

\section{A. Numerical Noise}

For the correct extraction and a high dynamic range of $S$-parameters on a transmission line, it might be necessary to use a discretization that is much smaller than the commonly used $\lambda / 10$ resolution since smaller cells reduce the numerical noise of a simulation. Smaller cells result in higher computational cost regarding memory and CPU time. This can be partly compensated by applying a local time-stepping scheme. However, to further increase efficiency, it is desirable to minimize the number of cells in a model, and a tradeoff between dynamic range and simulation time has to be found. A way to increase the dynamic range of the scattering parameters without decreasing the mesh size in the model is the use of mode projection. An example of the computation of $S$-parameters with and without mode projection in a coaxial torus resonator is shown in Section VII.

\section{B. Multimode Environment}

In a multimode environment, each electromagnetic mode on a physical port is assigned to one electromagnetic port $p$. After separating the total field into an outgoing and incoming part following the previously described approach of wave separation, the amplitudes $A_{p}^{ \pm}$of the incident and reflected waves of mode $p$ can be determined using (18) or (20). With these mode amplitudes, the incoming $a$ and outgoing $b$ waves in a port can be computed in order to extract the generalized scattering matrix.

\section{S-PARAMETER-EXTRACTION SCHEME}

Here, a novel scheme to perform a fully field-based approach to extract generalized scattering matrices is introduced. The extraction scheme is based on the flux-separation approach explained in Section IV. It can be applied in any numerical method that makes use of volume discretization, and it does not require the support from auxiliary transmission-line parameters.

For comparison, another approach using modal UIs on a transmission line is adapted to the FVTD technique and shown additionally in Section VI-C.

In the following, frequency-domain values, obtained by a Fourier transformation performed on the fly during the FVTD simulation, are denoted with a hat ${ }^{\smile}$.

\section{A. Full-Wave Approach for Guides With Homogeneous Cross Section}

For the extraction of scattering parameters, the tangentialfield distribution of the desired modes needs to be known in the port plane. The total electromagnetic field in the barycenters of the tetrahedral cells in the numerical model is obtained from the FVTD simulation. The total field in the port plane is then determined either: 1) from the adjacent tetrahedra using the MUSCL algorithm or 2) by a fitting procedure using the fields in the tetrahedra in a predefined neighborhood. Applying the FS scheme, the flux through the port can be separated into an outgoing $\boldsymbol{\Phi}_{i k}^{+}$ (14) and an incoming $\boldsymbol{\Phi}_{j k}^{-}$(15) part. With an adequate choice of the orientation of a port plane (in other words, the definition of the front and back sides of a port plane, as depicted in Fig. 1), the outgoing flux can unambiguously be connected to the incident wave $a$, and the incoming flux to the reflected wave $b$ in a port.

Since the FS scheme is based on plane waves traveling through the cell's faces in direction of the face's normal vector, the ratio between the electric and magnetic parts of the flux is given by the intrinsic impedance

$$
\frac{\breve{\Phi}_{x}^{E^{+}}}{\breve{\Phi}_{y}^{H^{+}}}=\eta=\sqrt{\frac{\mu}{\varepsilon}} .
$$

In (21), a guided wave with $\breve{E}_{y}$ and $\breve{H}_{x}$ components propagating in the negative $z$-direction is assumed. In contrast, the relation of the electric and magnetic fields of the same wave in the barycenter of a tetrahedral cell yields the wave impedance

$$
\frac{\breve{E}_{y}}{\breve{H}_{x}}=Z_{p}
$$

For a TEM mode, (21) and (22) both deliver the same impedance $Z_{p}=\eta$. In a non-TEM waveguide, however, this is not the case and the separated fluxes do not yield proper power waves in a port plane. To assure correct results for the fluxes in the port, the flux matrix given in (13) needs to be corrected locally. This correction is only performed in the frame of the extraction of the scattering parameters and does not affect the general algorithm of the electromagnetic field in the computational domain.

Consider the same wave as investigated above, and a port plane (normal vector $\boldsymbol{n}=(0,0,-1)^{T}$ ) that is oriented perpendicular to the propagation direction. The outgoing and incoming flux for the electric field using (11)-(13) can be written as

$$
\begin{aligned}
& \breve{\Phi}_{x}^{E^{+}}=\frac{1}{2 \mu} \breve{E}_{y}+\frac{c}{2} \breve{H}_{x}=\frac{\breve{E}_{y}}{2 \mu}\left(1+\eta \frac{\breve{H}_{x}}{\breve{E}_{y}}\right) \\
& \breve{\Phi}_{x}^{E^{-}}=\frac{1}{2 \mu} \breve{E}_{y}-\frac{c}{2} \breve{H}_{x}=\frac{\breve{E}_{y}}{2 \mu}\left(1-\eta \frac{\breve{H}_{x}}{\breve{E}_{y}}\right) .
\end{aligned}
$$

The incident and reflected waves in a port associated with (23) and (24) do not yield the correct power density in a port. To assure the correct computation of the tangential fields in a port, a mode-dependent wave velocity $c_{e}^{\prime}=1 / \sqrt{\varepsilon^{\prime} \mu}$ with the permittivity $\varepsilon^{\prime}=\varepsilon / \xi^{2}$ has to be introduced. 
Depending on the considered mode of the electromagnetic wave, the modified velocity $c_{e}^{\prime}$ is characterized by

$$
c_{e}^{\prime}=\frac{\xi}{\sqrt{\varepsilon \mu}} \begin{cases}\text { 1. TEM Mode } & \xi=1 \\ 2 . \text { TE Mode } & \xi=1 / \sqrt{1-\left(\frac{f_{c}}{f_{0}}\right)^{2}} \\ 3 . \text { TM Mode } & \xi=\sqrt{1-\left(\frac{f_{c}}{f_{0}}\right)^{2}}\end{cases}
$$

where $f_{c}$ is the cutoff frequency of the corresponding mode that is known a priori from the mode templates.

Inserting $c_{e}^{\prime}$ in the lower three lines of (13) yields a modified flux $\breve{\varphi}$

$$
\begin{aligned}
& \breve{\varphi}_{x}^{E^{+}}=\frac{1}{2 \mu} \breve{E}_{y}+\frac{c_{e}^{\prime}}{2} \breve{H}_{x}=\frac{\breve{E}_{y}}{2 \mu}\left(1+\xi \eta \frac{\breve{H}_{x}}{\breve{E}_{y}}\right) \\
& \breve{\varphi}_{x}^{E^{-}}=\frac{1}{2 \mu} \breve{E}_{y}-\frac{c_{e}^{\prime}}{2} \breve{H}_{x}=\frac{\breve{E}_{y}}{2 \mu}\left(1-\xi \eta \frac{\breve{H}_{x}}{\breve{E}_{y}}\right) .
\end{aligned}
$$

The considered electromagnetic wave may consist of an incident $i$ and a reflected $r$ part $\breve{E}_{y}=\breve{E}_{y}^{i}+\breve{E}_{y}^{r}=(1+\Gamma) \breve{E}_{y}^{i}$ and $\breve{H}_{x}=(1-\Gamma) \breve{H}_{x}^{i}$, respectively. Thus, the outgoing and incoming fluxes can be written as

$$
\begin{aligned}
& \breve{\varphi}_{x}^{E^{+}}=\frac{\breve{E}_{y}^{i}}{2 \mu}\left[\left(1+\xi \eta \frac{\breve{H}_{x}}{\breve{E}_{y}}\right)+\Gamma\left(1-\xi \eta \frac{\breve{H}_{x}}{\breve{E}_{y}}\right)\right] \\
& \breve{\varphi}_{x}^{E^{-}}=\frac{\breve{E}_{y}^{i}}{2 \mu}\left[\left(1-\xi \eta \frac{\breve{H}_{x}}{\breve{E}_{y}}\right)+\Gamma\left(1+\xi \eta \frac{\breve{H}_{x}}{\breve{E}_{y}}\right)\right] .
\end{aligned}
$$

With the parameter $\xi$ defined in (25), the fluxes in (28) and (29) yield

$$
\breve{\varphi}_{x}^{E^{+}}=\frac{\breve{E}_{y}^{i}}{\mu} \text { and } \breve{\varphi}_{x}^{E^{-}}=\Gamma \frac{\breve{E}_{y}^{i}}{\mu} .
$$

If the computation of the magnetic part of the flux is desired, a corresponding modified velocity $c_{h}^{\prime}=1 / \sqrt{\varepsilon \mu^{\prime}}$ with the permeability $\mu^{\prime}=\mu \cdot \xi^{2}$ has to be introduced in the upper three lines of (13).

Since the fluxes are connected to the tangential fields in the port, these fields can now be retrieved as follows:

$$
\begin{aligned}
& \breve{\boldsymbol{E}}_{t}^{ \pm}=-\boldsymbol{n} \times \mu \breve{\boldsymbol{\varphi}}^{E^{ \pm}} \\
& \breve{\boldsymbol{H}}_{t}^{ \pm}=\boldsymbol{n} \times \varepsilon \breve{\varphi}^{H^{ \pm}} .
\end{aligned}
$$

Equation (30) together with (31) and (32) deliver the correct result for the tangential fields in the port plane. This can be proven by determining the power density of the incident wave

$$
\breve{S}^{i}=\frac{1}{2}\left(\breve{E}_{t}^{+} \times \breve{\boldsymbol{H}}_{t}^{+*}\right)=\frac{1}{2} \frac{\left|\breve{E}_{y}^{i}\right|^{2}}{Z_{p}} .
$$

The power density of the reflected wave can be verified accordingly.

Now that the incident $(+)$ and reflected $(-)$ tangential electric fields in the port plane are determined, they can be disassembled into single modes, as explained in Section V. Applying the mode projection with the help of the mode-template vector $\boldsymbol{e}_{p}^{\prime}$, the mode amplitude of the incident and reflected waves of mode $p$ can be determined as follows:

$$
\breve{A}_{p}^{\prime \pm}=\sum_{k=1}^{K}\left(\breve{\boldsymbol{E}}_{t}^{ \pm}(k) \cdot \boldsymbol{e}_{p}^{\prime}(k)\right) F_{k} .
$$

The incident and reflected power waves $\breve{a}_{p}$ and $\breve{b}_{p}$ for mode $p$, delivering the information of the incident and reflected power in a port plane, are defined as

$$
\breve{a}_{p}=\frac{\breve{A}_{p}^{\prime+}}{\sqrt{Z_{p}}} \quad \breve{b}_{p}=\frac{\breve{A}_{p}^{\prime-}}{\sqrt{Z_{p}}}
$$

where $Z_{p}$ is the wave impedance of the electromagnetic field on the transmission line.

For this pure field-based approach of $S$-parameter extraction, the following a priori information is needed for each mode:

- tangential mode-template vector $\boldsymbol{e}_{p}^{\prime}$;

- propagation constant $\beta$.

Using this information together with the tangential fields (computed with any numerical method) in the port plane, the scattering parameters can be extracted.

\section{B. Extension to Guides With Inhomogeneous Cross Section}

In the case of inhomogeneous guides, the correction factor $\xi$ introduced in Section VI-A has to be found in a generalized manner. Depending on the current field components, the correction factor $\xi_{(k)}$ at location $k$ is characterized by

$$
\begin{aligned}
& \xi_{e x}(k)=\frac{e_{y p}^{\prime \prime}(k)}{h_{x p}^{\prime \prime}(k)} \cdot \eta^{-1} \\
& \xi_{e y}(k)=-\frac{e_{x p}^{\prime \prime}(k)}{h_{y p}^{\prime \prime}(k)} \cdot \eta^{-1}
\end{aligned}
$$

These parameters have to be introduced in (13) (as explained in Section VI-A) to compute the flux for the electric field. The appropriate column in (13) has to be chosen in order to multiply the correction factor with the adequate magnetic field (i.e., the same index of correction factor and magnetic field). The mode amplitude is determined by

$$
\breve{A}_{p}^{\prime \prime+/-}=\sum_{k=1}^{K}\left(\breve{\boldsymbol{E}}_{t}^{ \pm}(k) \times \breve{\boldsymbol{h}}_{p}^{\prime \prime}(k)\right) \boldsymbol{F}_{k} .
$$

The change of the transverse-field distributions $\boldsymbol{e}_{p}^{\prime \prime}$ and $\boldsymbol{h}_{p}^{\prime \prime}$ (mode template vectors) with frequency in inhomogeneous guides can be accounted for by using multiple mode template vectors at different sample frequencies. However, the mode 
template vectors do not change drastically with frequency. Thus, it is usually sufficient to employ only one template in (38) at a certain frequency [7]. An example of a simulation of an inhomogeneous guide can be found in Section VII-D where the $S$-parameter computation of a rectangular waveguide filter loaded with dielectric slabs is presented.

\section{Transmission-Line Approach}

The concept of wave separation using power waves on the basis of UI was introduced by Kurokawa in 1965 [15] where the power of incident $a_{p}$ and reflected $b_{p}$ waves of a certain mode $p$ are defined by

$$
\breve{a}_{p}=\frac{\breve{U}_{p}+Z_{p} \breve{I}_{p}}{2 \sqrt{\left|\operatorname{Re}\left\{Z_{p}\right\}\right|}} \quad \breve{b}_{p}=\frac{\breve{U}_{p}-Z_{p} \breve{I}_{p}}{2 \sqrt{\left|\operatorname{Re}\left\{Z_{p}\right\}\right|}} .
$$

The square of the magnitude of the power waves determine the incident and the reflected power on a transmission line. To calculate these power waves, the mode voltage $\breve{U}_{p}$ and current $\breve{I}_{p}$ and the impedance $Z_{p}$ have to be determined, resulting from an integration over the tangential electric $\boldsymbol{E}_{t p}$ and magnetic $\boldsymbol{H}_{t p}$ field in the port plane. These path integrals are only unambiguously determined in TEM structures.

An extension to this approach, using integral quantities, is presented in [7] by Gwarek and Celuch-Marcysiak. In this scheme, modal UI on a port are defined as

$$
\begin{aligned}
U_{p}(t) & =\sum_{k=1}^{K}\left(\boldsymbol{E}(k, t) \times \boldsymbol{h}_{p}^{\prime \prime}(k)\right) \boldsymbol{F}_{k} \\
I_{p}(t) & =\sum_{k=1}^{K}\left(\boldsymbol{e}_{p}^{\prime \prime}(k) \times \boldsymbol{H}(k, t)\right) \boldsymbol{F}_{k}
\end{aligned}
$$

where $\boldsymbol{e}_{p}^{\prime \prime}$ is the electric and $\boldsymbol{h}_{p}^{\prime \prime}$ is the magnetic mode-template vector (the same as introduced in Section V). Therefore, this approach is applicable to inhomogeneous guides. For the extraction of a generalized scattering matrix, the following definitions of the incident and reflected waves are used:

$$
\breve{a}_{p}=\frac{\breve{U}_{p}+Z_{p} \breve{I}_{p}}{2 \sqrt{Z_{p}}} \quad \breve{b}_{p}=\frac{\breve{U}_{p}-Z_{p} \breve{I}_{p}}{2 \sqrt{Z_{p}}}
$$

The impedance $Z_{p}$ is calculated with

$$
Z_{p}=\sqrt{\frac{\breve{U}_{p} \cdot \partial \breve{U}_{p} / \partial \boldsymbol{n}}{\breve{I}_{p} \cdot \partial \breve{I}_{p} / \partial \boldsymbol{n}}}
$$

where the derivatives along the propagation direction (denoted by $\partial / \partial \boldsymbol{n}$ ) of voltage and current are determined using a standard finite-differencing scheme. In the unstructured FVTD mesh, an interpolated port plane is introduced for the computation of modal voltages, currents, and their derivatives. To enable the use of finite differencing, two auxiliary interpolation planes (similar to the one described in Section III-B) are required in contrast to just one plane needed for the scheme proposed in

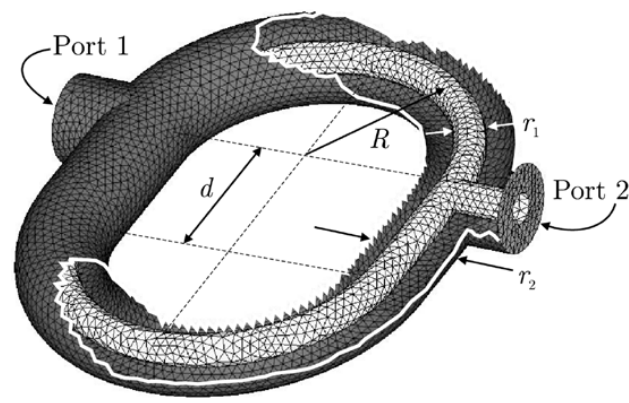

Fig. 4. Cut-open geometry of a coaxial torus. Only the triangular surface mesh is shown.

Section VI-A. The two port planes are chosen equally spaced on both sides of the port plane in a distance of a fraction of the wavelength. The electromagnetic-field values on the actual port plane are determined from the values located on the auxiliary planes through arithmetic averaging.

\section{EXAMPLES}

The extraction of scattering parameters using the proposed method is shown for TEM and non-TEM waveguide problems. In all examples, the results obtained by the FVTD method using the generalized scattering-parameter extraction scheme introduced in Section VI are successfully compared to reference solutions obtained by CST Microwave Studio (MWS) and/or a mode-matching (MM) approach. Additional examples of successful $S$-parameter extractions using the FVTD method can be found in [5] and [16].

\section{A. Coaxial-Torus Resonator}

The TEM coaxial-torus resonator is fed by a coaxial line with an inner conductor radius of $r_{1}=1.25 \mathrm{~mm}$ and an outer radius of $r_{2}=3.4 \mathrm{~mm}$. The permittivity of the dielectric material is $\varepsilon_{r}=2.2$. The bending radius of the torus, as shown in Fig. 4 , is $R=40 / \pi \mathrm{mm}$. In the plane, where both coaxial ports are located, the torus is extended by an additional length of $d=10 \mathrm{~mm}$. The partly cut-open geometry including the triangular surface mesh is depicted in Fig. 4. The tetrahedral volume mesh is not shown for a better visibility of the geometry. In this figure, the inner and outer conductor and the two boundary port planes are apparent. A Gaussian pulse covering a bandwidth from $f=0.01 \mathrm{GHz}$ to $f=4.5 \mathrm{GHz}$ is used for the excitation of port 1 . The coaxial-torus resonator shows two resonances in this band at $f_{r 1}=2.01 \mathrm{GHz}$ and $f_{r 2}=4.02 \mathrm{GHz}$. The reflection and transmission coefficients are plotted in Fig. 5. Results obtained by the FVTD's FS scheme are compared to a reference MWS solution, showing a very good agreement over the whole frequency range. The advantage to apply a mode-projection algorithm even in a TEM mode environment can be seen in Fig. 6 where a zoom in the second resonance of the coaxial torus is depicted. The solid lines represents the result of $\left|S_{21}\right|$ obtained by a simulation making use of mode projection and the dashed line depicts the results of the transmission coefficient obtained without a mode-projection scheme. Both results are obtained with the same mesh illustrating that mode projection enhances the dynamic range of extracted $S$-parameters. 


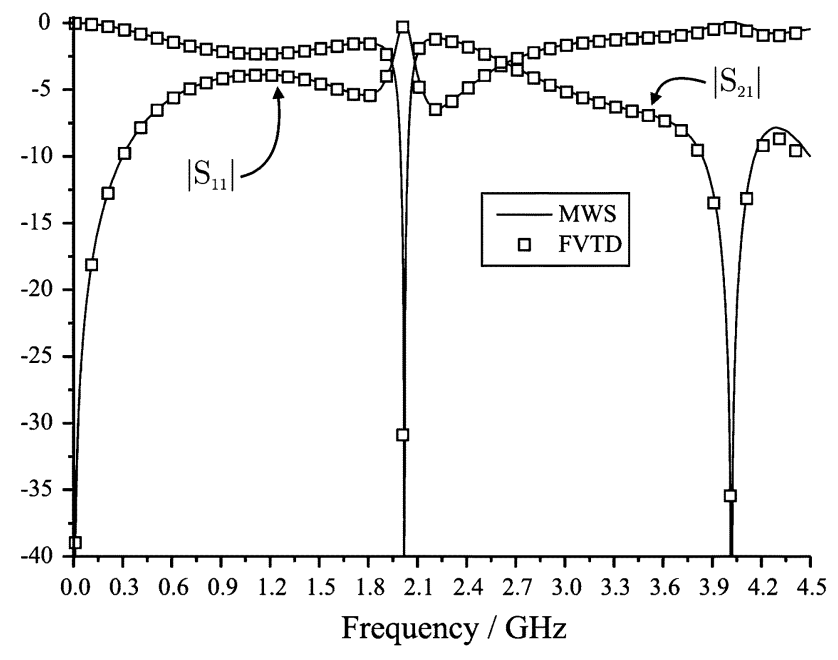

Fig. 5. Reflection and transmission coefficient of the coaxial torus. The results of the FVTD simulation are compared to MWS results used as a reference.

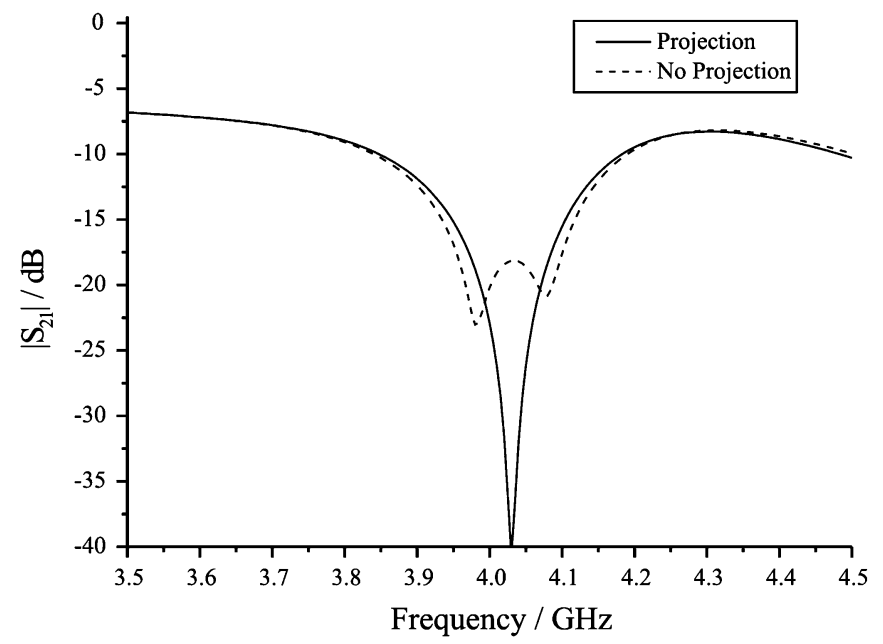

Fig. 6. Magnification of transmission coefficient of the second resonance of the coaxial torus obtained with FVTD.

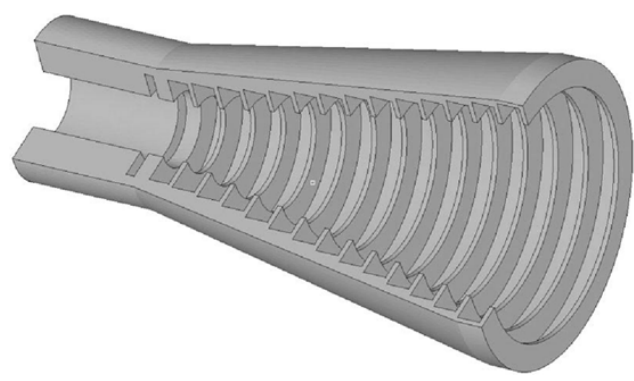

Fig. 7. Cut-open geometry of a conical corrugated horn antenna.

\section{B. Conical Corrugated Horn Antenna}

The second example is a conical corrugated horn antenna exhibiting a very low cross-polarization level. The simulation of such an antenna is a demanding task since a high dynamic range is required in the numerical simulation to yield accurate results. The cut-open geometry of a conical corrugated horn is illustrated in Fig. 7. The design includes 15 corrugations, the first three of which constitute a $\mathrm{TE}_{11}$-to- $\mathrm{HE}_{11}$-mode converter, while the remaining corrugations support the converted mode

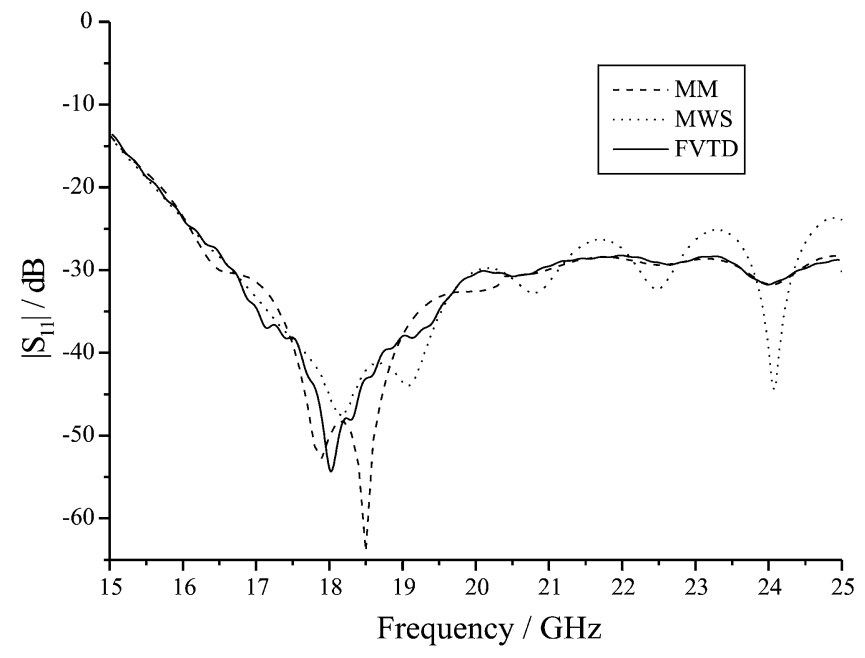

Fig. 8. Return loss of the conical corrugated horn antenna. The graph depicts a comparison between results obtained by an MM approach (dashed line), by MWS (dotted line), and by FVTD (solid line).

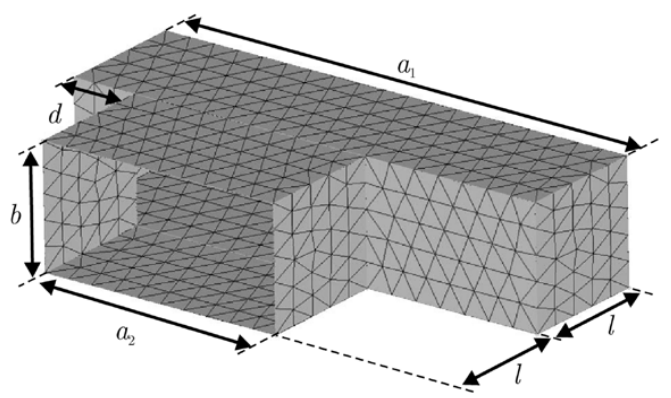

Fig. 9. Geometry of the $H$-plane step discontinuity. Only the triangular surface mesh is shown. The port planes are located in a distance $l=1.5 \mathrm{~mm}$ from the discontinuity.

along the horn length. In the design frequency band, the electric and magnetic fields are balanced in order to produce both pattern symmetry and low cross-polarization.

The return loss of the corrugated horn antenna is plotted in Fig. 8. Results from an FVTD simulation (solid line) using the presented scheme are compared to results obtained by MWS (dotted line) and by an MM approach (dashed line). All three results exhibit a good agreement in the whole frequency band.

\section{H-Plane Step Discontinuity}

The scattering parameters of a rectangular waveguide $H$-plane step discontinuity are computed using both the FS procedure and the scheme using modal UIs. An MM analysis yields a reference solution.

The geometry including the meshed surface is depicted in Fig. 9. The $H$-plane step discontinuity consists of a transition from a waveguide with the dimension $a_{1}=38.1 \mathrm{~mm}$ and $b=$ $9.525 \mathrm{~mm}$ to a WR75 waveguide with half the width $a_{2}=$ $19.05 \mathrm{~mm}$ and the same height $b$. The transition is asymmetrical, the WR75 waveguide being displaced by $d=5 \mathrm{~mm}$ with regard to the first waveguide. The port planes are located at the same distance on both sides of the discontinuity $(l=1.5 \mathrm{~mm})$. A Gaussian pulse covering the necessary bandwidth is used for exciting the fundamental $\mathrm{TE}_{10}$ mode in the first waveguide. 


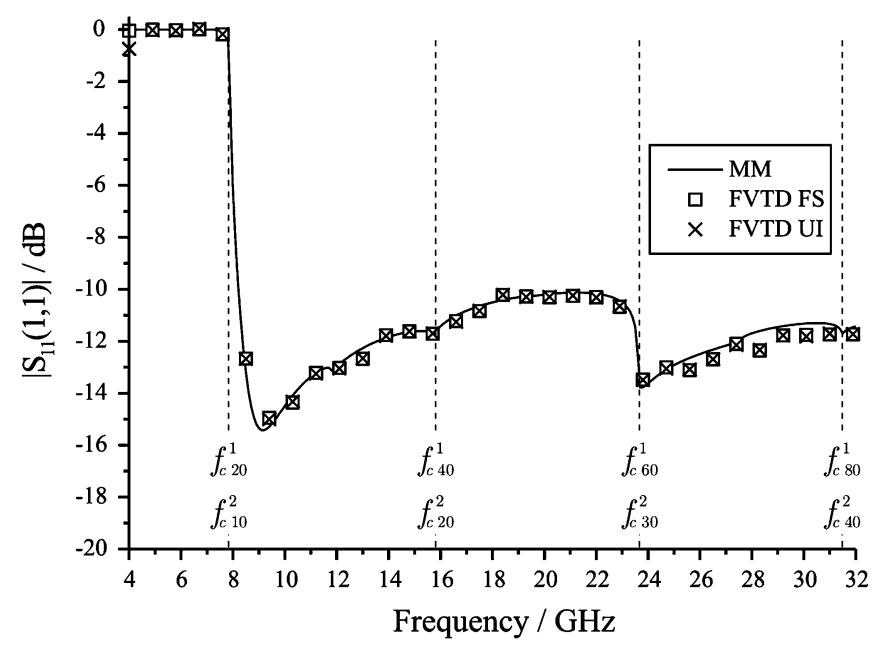

Fig. 10. Comparison between FVTD and MM results of the reflection coefficient $\left|S_{11}(1,1)\right|$ for the fundamental mode at the $H$-plane step discontinuity.

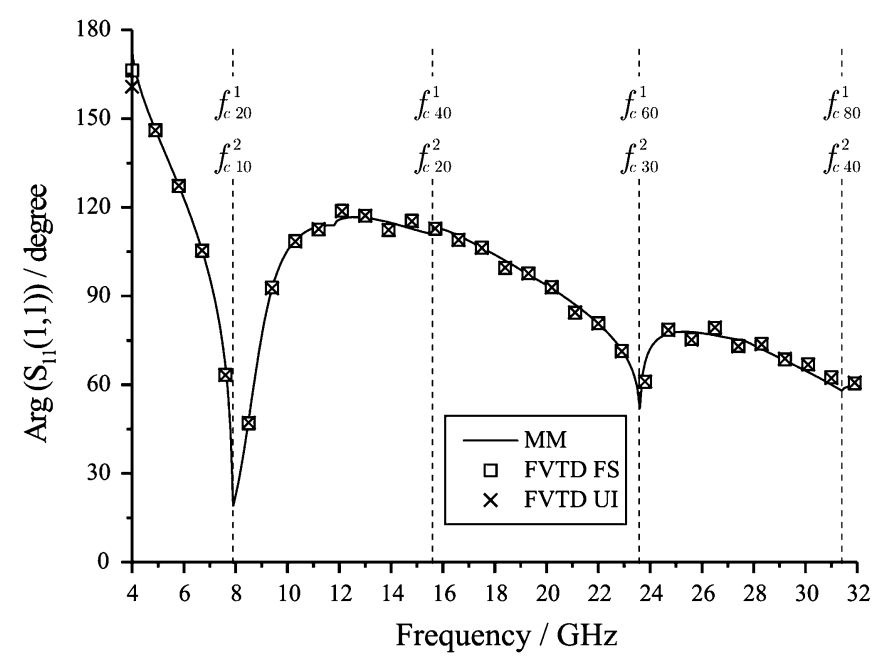

Fig. 11. Comparison between FVTD and MM results of the phase of the reflection coefficient $S_{11}(1,1)$ for the fundamental mode at the $H$-plane step discontinuity.

The reflection coefficient $\left|S_{11}(1,1)\right|$ for the fundamental mode is presented in Fig. 10. The solid line displays the MM analysis, and the squares and crosses show the results obtained from the FVTD analysis using the FS and UI scheme, respectively. Both FS and UI extraction approaches deliver very similar results and both are in agreement with the reference solution. This is also the case for the phase of $S_{11}(1,1)$ (Fig. 11), which is very sensitive to simulation inaccuracies. While the previous investigation was focused on the fundamental mode only, the following also takes into account higher order modes. Here, the mode-projection method is used to generate the generalized scattering matrix. Fig. 12 depicts the reflection coefficient $\left|S_{11}(2,1)\right|$ from the incident fundamental mode to the reflected $\mathrm{TE}_{20}$ mode. Again, the agreement between the FVTD results and MM analysis is good. The reflection coefficient $\left|S_{11}(3,1)\right|$ from the incident $\mathrm{TE}_{10}$ to $\mathrm{TE}_{30}$ modes also shows a good agreement (Fig. 13). The transmission coefficient $\left|S_{21}(1,1)\right|$ for the fundamental mode is plotted in Fig. 14 . The result demonstrates that the use of the FS scheme yields

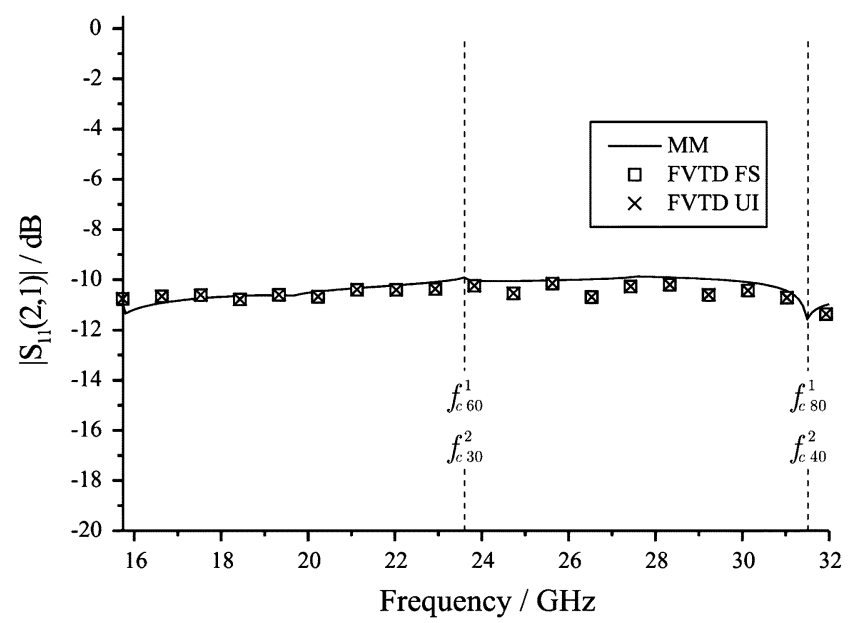

Fig. 12. Comparison between FVTD and MM results of the reflection coefficient $\left|S_{11}(2,1)\right|$ from incident $\mathrm{TE}_{10}$ into reflected $\mathrm{TE}_{20}$ mode at the $H$-plane step discontinuity.

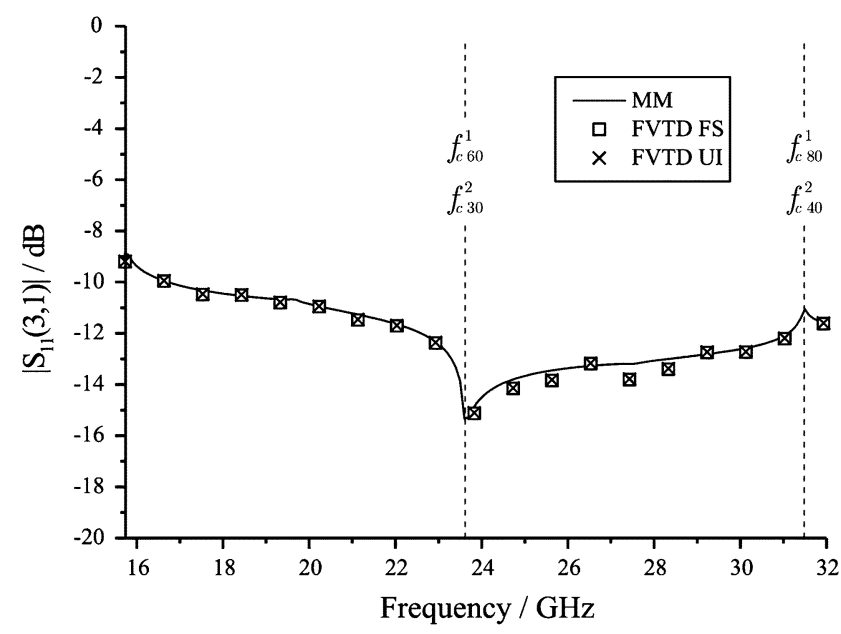

Fig. 13. Comparison between FVTD and results of the reflection coefficient $\left|S_{11}(3,1)\right|$ from incident $\mathrm{TE}_{10}$ into reflected $\mathrm{TE}_{30}$ mode at the $H$-plane step discontinuity.

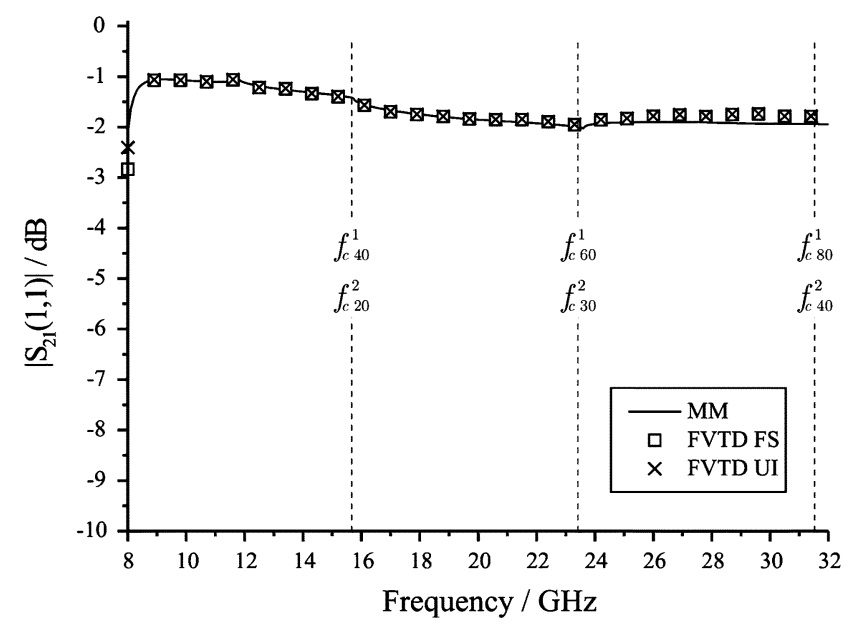

Fig. 14. Comparison between FVTD and MM results of the transmission coefficient $\left|S_{21}(1,1)\right|$ for the fundamental mode at the $H$-plane step discontinuity.

the correct power in a port plane, as explained in Section VI. Figs. 15 and 16 depict the transmission coefficients $\left|S_{21}(2,1)\right|$ and $\left|S_{21}(3,1)\right|$ from the fundament mode to $\mathrm{TE}_{20}$, and $\mathrm{TE}_{30}$, respectively. 


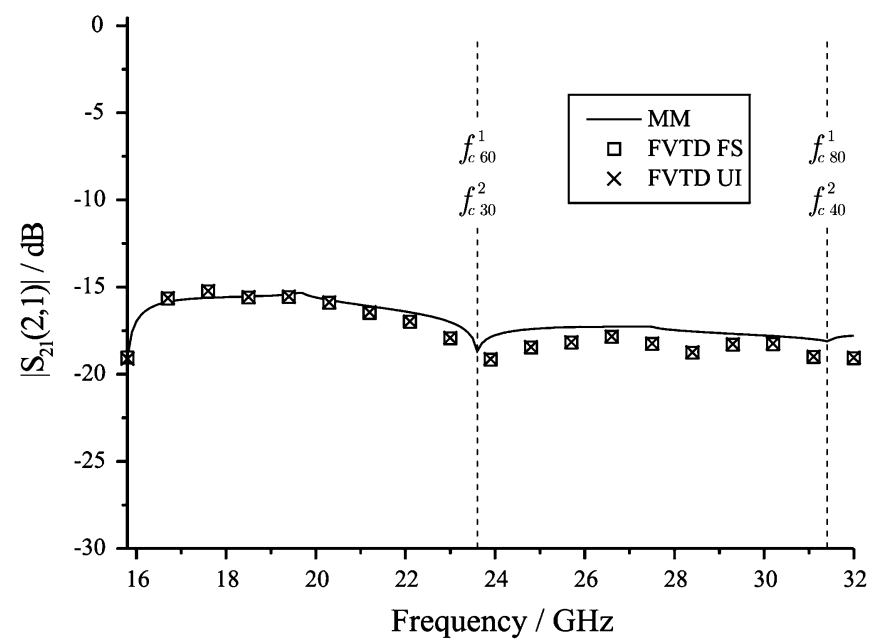

Fig. 15. Comparison between FVTD and MM results of the transmission coefficient $\left|S_{21}(2,1)\right|$ from incident $\mathrm{TE}_{10}$ into transmitted $\mathrm{TE}_{20}$ mode at the $H$-plane step discontinuity.

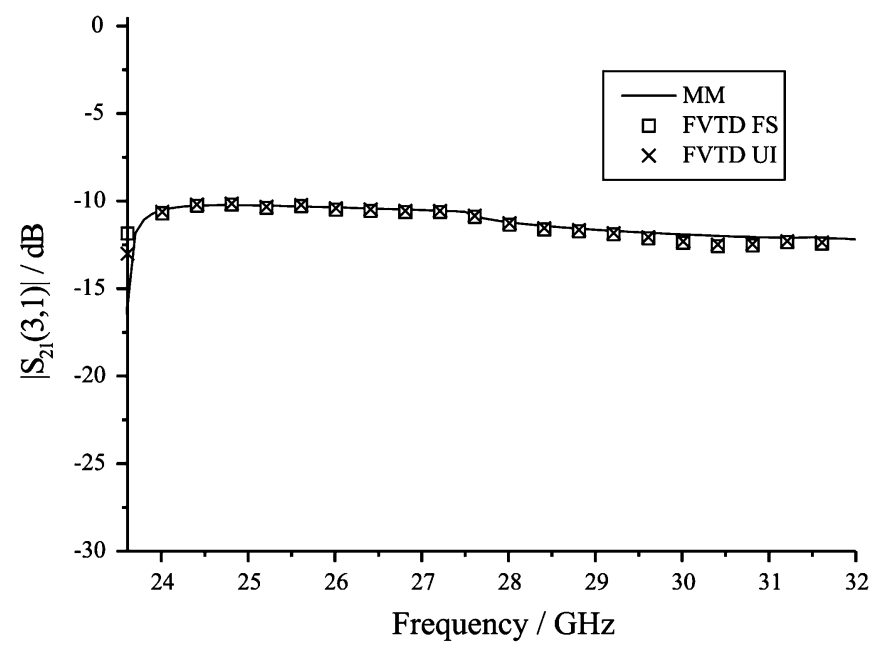

Fig. 16. Comparison between FVTD and MM results of the transmission coefficient $\left|S_{21}(3,1)\right|$ from incident $\mathrm{TE}_{10}$ into transmitted $\mathrm{TE}_{30}$ mode at the $H$-plane step discontinuity.

\section{Inhomogeneously Filled Waveguide}

As an example of a guide having an inhomogeneous cross section, a rectangular waveguide filter is chosen. Such waveguides partially filled with dielectric slabs are employed in a variety of components and are comprehensively investigated in literature [17]-[20]. The structure investigated here is taken from [7], where it also serves as a testing structure for the correct extraction of $S$-parameters in inhomogeneous guides. Fig. 17 shows the cut-open geometry of the waveguide filter. The surface triangulation is not shown for a better visibility. The dimensions of the waveguide are $a=5 \mathrm{~mm}$ and $b=2 \mathrm{~mm}$. The filter incorporates four dielectric slabs that are centered with regard to $a$ and that extend over the full height $b$ of the waveguide. The filter is symmetric with respect to the longitudinal center, and all slabs have the same width $t=1 \mathrm{~mm}$. At the input port 1 and the output port 2, the dielectric slabs exhibit a relative permittivity of $\varepsilon_{r 1}=9.7$. The displacement to the next dielectric is $d_{1}=2 \mathrm{~mm}$. The two slabs located in the middle of the filter (denoted with a light gray color in Fig. 17) are $l=2 \mathrm{~mm}$ long

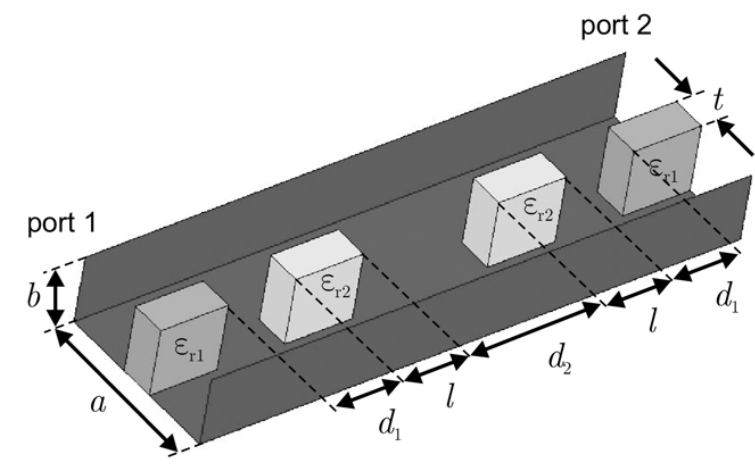

Fig. 17. Cut-open geometry of the waveguide filter [7]. The triangulation of the surfaces is not shown. The distance between ports 1 and 2 is $14 \mathrm{~mm}$.

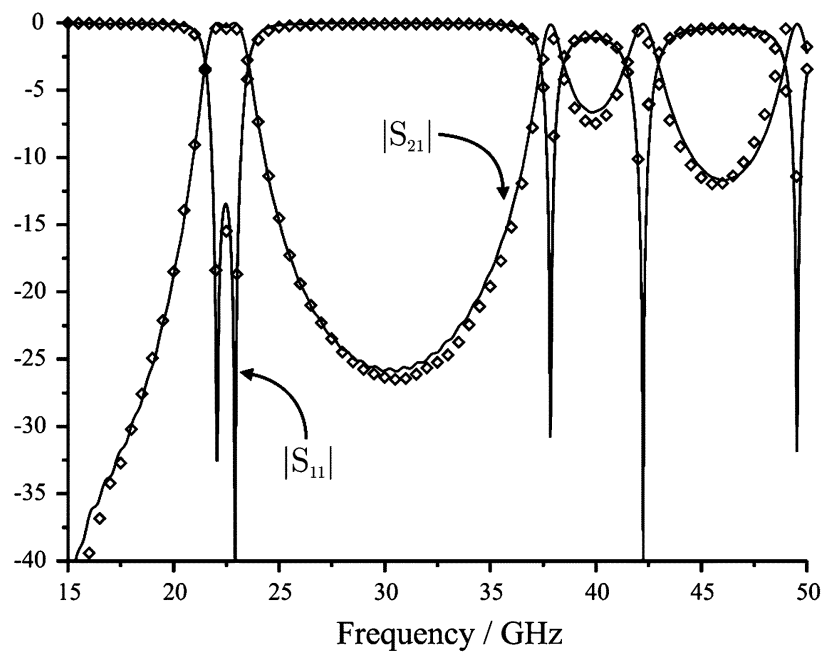

Fig. 18. Reflection $\left|S_{11}\right|$ and transmission $\left|S_{21}\right|$ coefficient of the waveguide filter with an inhomogeneous cross section. The results of the FVTD simulation are compared to results obtained by MWS.

and have a relative permittivity of $\varepsilon_{r 2}=7$. The distance between these two dielectrics is $d_{2}=4 \mathrm{~mm}$. The input port 1 and output port 2 are equidistantly displaced from the filter center by $7 \mathrm{~mm}$.

Fig. 18 presents the scattering parameters of the waveguide filter. Results of the FVTD simulation are compared to results obtained by an MWS simulation, which are used as a reference solution. A good agreement is found for the whole frequency range, showing that the proposed method is also applicable in inhomogeneous structures.

\section{CONCLUSION}

A novel field-based scheme for the extraction of generalized scattering parameters has been presented. The proposed method has exploited the flux-separation algorithm that originates from finite-volume techniques. Since all required parameters and the employed FS scheme are already known and naturally included in the FVTD algorithm, this scheme is advantageously applicable in the scope of FVTD simulations. However, the scheme can be applied to any volume-discretizing numerical method both in the time and frequency domains. Since the flux-separation is based on a plane-wave assumption, modified velocities for the electric and magnetic fields have to be introduced 
in the FS matrix used for the $S$-parameter extraction to assure correct power flows through the port planes. This correction does not affect the general numerical algorithm since it is only used in the frame of $S$-parameter extraction. Four examples, i.e., a coaxial-torus resonator, a conical corrugated horn, a rectangular waveguide discontinuity, and an inhomogeneously loaded waveguide filter, have illustrated the accuracy of the proposed scheme.

\section{ACKNOWLEDGMENT}

The authors would like to thank M. Gimersky, E. Ofli, G. Tudosie, and K. Krohne, all with the Laboratory for Electromagnetic Fields and Microwave Electronics, Swiss Federal Institute of Technology Zürich, Zürich, Switzerland, for the computation of the reference solutions.

\section{REFERENCES}

[1] P. Bonnet, X. Ferrieres, F. Paladian, J. Grando, J. Alliot, and J. Fontaine, "Electromagnetic wave diffraction using a finite volume method," Electron Lett., vol. 33, no. 1, pp. 31-32, 1997.

[2] C. Fumeaux, D. Baumann, and R. Vahldieck, "Advanced FVTD simulations of dielectric resonator antennas and feed structures," in ACES J., vol. 19, 2004, pp. 155-164.

[3] D. Baumann, C. Fumeaux, P. Leuchtmann, and R. Vahldieck, "Finitevolume time-domain (FVTD) modeling of a broad-band double-ridged horn antenna," Int. J. Numer. Modeling, vol. 17, no. 3, pp. 285-298, 2004.

[4] V. Shankar, A. H. Mohammadian, and W. F. Hall, "A time-domain, finite-volume treatment for the maxwell equations," Electromagnetics, vol. 10, pp. 127-145, 1990.

[5] D. Baumann, C. Fumeaux, and R. Vahldieck, "A novel wave-separation scheme for the extraction of $S$-parameters in non-TEM waveguides for the FVTD method," in IEEE MTT-S Int. Microwave Symp. Dig., Long Beach, CA, Jun. 2005. [CD ROM].

[6] P. Bonnet, X. Ferrieres, B. Michielsen, and P. Klotz, Time Domain Electromagnetics, S. M. Rao, Ed. New York: Academic, 1997, ch. 9: Finite-Volume Time Domain Method, pp. 307-367.

[7] W. K. Gwarek and M. Celuch-Marcysiak, "Wide-band $S$-parameter extraction from FD-TD simulations for propagating and evanescent modes in inhomogeneous guides," IEEE Trans. Microw. Theory Tech., vol. 51, no. 8, pp. 1920-1928, Aug. 2003.

[8] N. K. Madsen and R. W. Ziolkowski, "A three-dimensional modified finite volume technique for maxwell's equations," Electromagnetics, vol. 10, pp. 147-161, 1990.

[9] C. Fumeaux, D. Baumann, P. Leuchtmann, and R. Vahldieck, “A generalized local time-step scheme for efficient FVTD simulations in strongly inhomogeneous meshes," IEEE Trans. Microwave Theory Tech., vol. 52, no. 3, pp. 1067-1076, Mar. 2004

[10] I. Amidror, "Scattered data interpolation methods for electronic imaging systems: A survey," J. Electron. Imaging, vol. 11, no. 2, pp. 157-176, 2002.

[11] G. K. Grau, Quantenelektronik.. Braunschweig, Germany: Vieweg, 1978 .

[12] J. Ritter and F. Arndt, "Efficient FDTD/matrix-pencil method for the full-wave scattering parameter analysis of waveguide structures," IEEE Trans. Microw. Theory Tech., vol. 44, no. 12, pp. 2450-2456, Dec. 1996.

[13] R. E. Collin, Field Theory of Guided Waves. New York: IEEE Press, 1991

[14] M. Mrozowski, Guided Electromagnetic Waves. Taunton, Somerset, U.K.: Res. Studios Press, 1997, ch. 10.

[15] K. Kurokawa, "Power waves and the scattering matrix," IEEE Trans. Microw. Theory Tech., vol. MTT-13, no. 3, pp. 194-202, Mar. 1965.

[16] D. Baumann, C. Fumeaux, P. Leuchtmann, and R. Vahldieck, "Generalized-scattering-matrix extraction using the finite-volume time-domain (FVTD) method," in IEEE MTT-S Int. Microwave Symp. Dig., Fort Worth, TX, Jun. 2004, pp. 1701-1704.

[17] A. Berk, "Variational principles for electromagnetic resonators and waveguides," IEEE Trans. Antennas Propag., vol. AP-4, no. 2, pp. 104-111, Feb. 1956.

[18] R. Sheikh and M. Gunn, "Wave propagation in a rectangular waveguide inhomogeneously filled with semiconductors," IEEE Trans. Microw. Theory Tech., vol. MTT-19, no. 2, pp. 117-121, Feb. 1968.
[19] P. Vartanian, W. Ayres, and A. Helgesson, "Variational principles for electromagnetic resonators and waveguides," IEEE Trans. Microw. Theory Tech., vol. MTT-6, no. 2, pp. 215-222, Feb. 1958.

[20] F. E. Gardiol, "Higher-order modes in dielectrically loaded rectangular waveguides," IEEE Trans. Microw. Theory Tech., vol. MTT-16, no. 11, pp. 919-924, Nov. 1968.

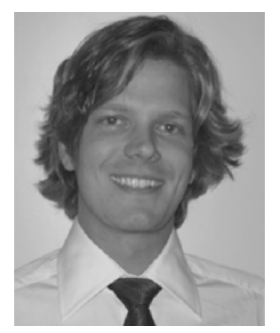

Dirk Baumann (S'01) received the Dipl.Ing. degree in electrical engineering from the University of Karlsruhe, Karlsruhe, Germany in 2001, and is currently working toward the Ph.D. degree in electrical engineering at the Swiss Federal Institute of Technology (ETH) Zürich, Zürich, Switzerland.

During the spring and fall of 2000 , he held an internship with the Alaska SAR Facility (ASF), Fairbanks, AK, where he was involved with the calibration of ASF's SAR processor. His research interests include numerical methods with emphasis on timedomain techniques and their application to general electromagnetic problems.

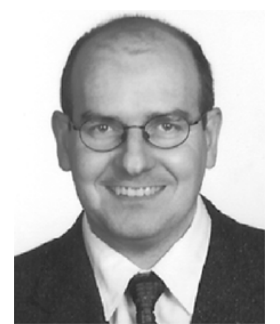

Christophe Fumeaux (M'03) received the Diploma and Ph.D. degrees in physics from the Swiss Federal Institute of Technology (ETH) Zürich, Switzerland, in 1992 and 1997, respectively. His doctoral dissertation concerned antenna-coupled infrared detectors

From 1998 to 2000, he was Post-Doctoral Researcher involved in infrared technology with the School of Optics, University of Central Florida, Orlando. In 2000, he joined the Swiss Federal Office of Metrology, Bern, Switzerland, as a Scientific Staff Member. Since 2001, he has been a Research Associate with the Laboratory for Electromagnetic Fields and Microwave Electronics (IFH), ETH Zürich, Switzerland. His current main research interest concerns computational electromagnetics in the time domain for numerical analysis of microwave circuits and antennas.

Dr. Fumeaux was the recipient of the ETH Silver Medal of Excellence for his doctoral dissertation.

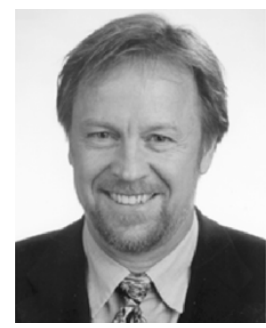

Rüdiger Vahldieck (M'85-SM'86-F'99) received the Dipl.-Ing. and Dr.-Ing. degrees in electrical engineering from the University of Bremen, Bremen, Germany, in 1980 and 1983, respectively.

From 1984 to 1986, he was a Post-Doctoral Fellow with the University of Ottawa, Ottawa, ON, Canada. In 1986, he joined the Department of Electrical and Computer Engineering, University of Victoria, Victoria, BC, Canada, where he became a Full Professor in 1991. During the fall of 1992 and the spring of 1993, he was a Visiting Scientist with the Ferdinand-Braun-Institute für Hochfrequenztechnik, Berlin, Germany. In 1997 , he accepted an appointment as a Professor of electromagnetic-field theory with the Swiss Federal Institute of Technology (ETH) Zürich, Zürich, Switzerland, and became Head of the Laboratory for Electromagnetic Fields and Microwave Electronics (IFH) in 2003. His research interests include computational electromagnetics in the general area of electromagnetic compatibility (EMC) and, in particular, for computer-aided design of microwave, millimeter-wave, and opto-electronic integrated circuits. Since 1981, he has authored or coauthored over 230 technical papers in books, journals, and conferences, mainly in the field of microwave computer-aided design.

Prof. Vahldieck is the past president of the IEEE 2000 International Zürich Seminar on Broadband Communications (IZS'2000). Since 2003, he has been president and general chairman of the International Zürich Symposium on Electromagnetic Compatibility. He is a member of the Editorial Board of the IEEE TRANSACTIONS ON MicrowaVe THEORY AND TECHNIQUes. From 2000 to 2003, he was an associate editor for the IEEE MiCROWAVE AND WIRELESS COMPONENTS LETTERS, and in January 2004, he became the editor-in-chief. Since 1992, he has served on the Technical Program Committee (TPC) of the IEEE Microwave Theory and Techniques Society (IEEE MTT-S) International Microwave Symposium (IMS), the IEEE MTT-S Technical Committee on Microwave Field Theory, and in 1999, on the TPC of the European Microwave Conference. From 1998 to 2003, he was the chapter chairman of the IEEE Swiss Joint Chapter on Microwave Theory and Techniques, Antennas and Propagation, and EMC. Since 2005, he has been president of the Research Foundation for Mobile Communications. He was the recipient of the J. K. Mitra Award of the Institution of Electronics and Telecommunication Engineers (IETE) (in 1996) for the best research paper in 1995 and was corecipient of the Outstanding Publication Award of the Institution of Electronic and Radio Engineers in 1983. 